\title{
Monitoring the kynurenine system: Concentrations, ratios or what else?
}

\author{
Masaru Tanaka ${ }^{\mathrm{A}-\mathrm{F}}$, László Vécsei ${ }^{\mathrm{A}-\mathrm{F}}$ \\ Department of Neurology, Interdisciplinary Excellence Centre, Faculty of Medicine, University of Szeged, Hungary \\ A - research concept and design; $B$ - collection and/or assembly of data; $C$ - data analysis and interpretation; \\ $D$ - writing the article; $E$ - critical revision of the article; $F$ - final approval of the article
}

Address for correspondence

Masaru Tanaka

E-mail: tanaka.masaru.1@med.u-szeged.hu

Funding sources

None declared

Conflict of interest

None declared

Received on June 22, 2021

Accepted on June 29, 2021

Published online on August 19, 2021

Cite as

Tanaka M, Vécsei L. Monitoring the kynurenine system: Concentrations, ratios or what else? Adv Clin Exp Med. 2021;30(8):775-778. doi:10.17219/acem/139572

D0I

10.17219/acem/139572

Copyright

Copyright by Author(s)

This is an article distributed under the terms of the

Creative Commons Attribution 3.0 Unported (CC BY 3.0)

(https://creativecommons.org/licenses/by/3.0/)

\begin{abstract}
The tryptophan-kynurenine metabolic pathway plays the most essential role in tryptophan metabolism, producing various endogenous bioactive molecules. The activation of the metabolic pathway is linked to the pathogenesis of a wide range of diseases. The calibration of the levels and the ratio of kynurenines has been attempted in search of biomarkers and diagnostic targets. This editorial introduces biosystems in close interaction with the kynurenine system and potential measures to assess a state of stress, which may lead to illnesses.
\end{abstract}

Key words: tryptophan, kynurenine, stress, neurological diseases, psychiatric diseases

\section{Introduction}

L-tryptophan (Trp) is the largest of the essential amino acids, the least abundant in the cell and the rarest in the proteome, which consumes the highest amount of energy in its biosynthesis among the 20 amino acids. ${ }^{1}$ It is an essential component in the biosynthesis of proteins, muscle and enzymes, as well as an obligatory substrate for the production of neurotransmitters and hormones. ${ }^{2}$ Acute Trp depletion causes increased pain sensitivity, acoustic startle, increased motor activity, and aggression, while chronic Trp deficiency causes ataxia, cognitive impairment and dysphoria with skin hyperpigmentation. ${ }^{3,4}$ The Trp is broken down through the methoxyindole pathway leading to the production of serotonin (5-hydroxytryptamine) or through the kynurenine (KYN) pathway leading to the de novo synthesis of nicotinamide adenine dinucleotide (NAD+). The KYN metabolic pathway consumes nearly 95\% of Trp, being the controller of Trp metabolism while producing a wide range of multifarious and versatile bioactive molecules. ${ }^{5}$ The molecules exhibit oxidant, antioxidant, anti-inflammatory, neurotoxin, neuroprotectant, and/or immunomodulating activity, but their actions depend on their concentrations and the cellular environment (Fig. 1). ${ }^{6}$ Furthermore, the KYN system works in close interaction with other biosystems, including the oxidative stress complex, the antioxidant system, serotonin neurotransmission, glutamate neurotransmission, the tetrahydrobiopterin pathway, the cannabinoid system, the $\mathrm{NAD}^{+} / \mathrm{NADH}$ redox system, and aryl hydrocarbon receptor (AHR) signaling. ${ }^{2,5}$ The accelerated Trp metabolism is generally observed in clinical conditions such as infection, inflammation, cancer, aging, neurodegenerative diseases, and psychiatric disorders. ${ }^{2,5,6,7-9}$ 


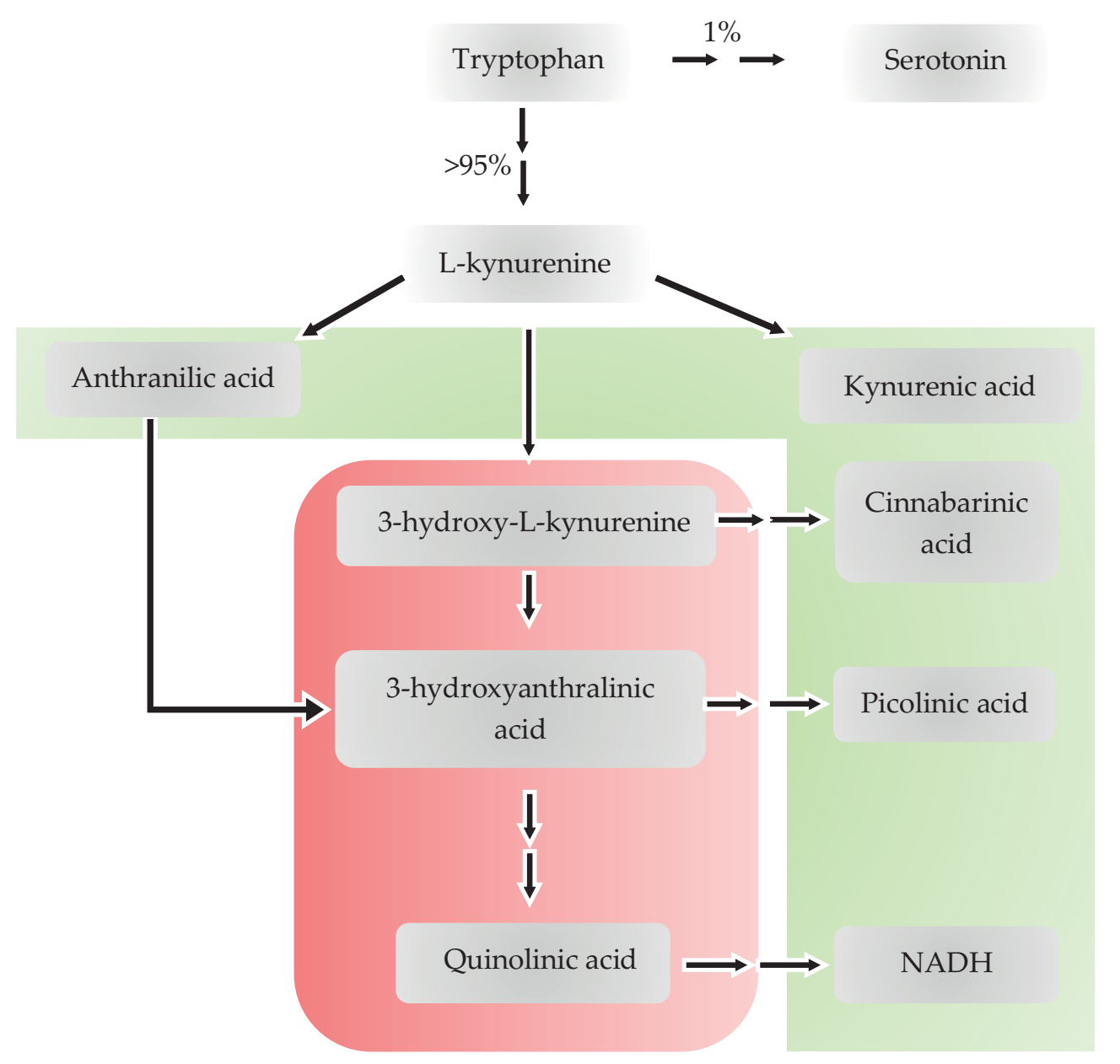

Fig. 1. Tryptophan (Trp) catabolism. More than 95\% of Trp is broken down through the kynurenine (KYN) pathway, while only $1 \%$ of Trp is through the methoxyindole pathway. Protective KYN metabolites are marked green and toxic KYN metabolites are imarked red. The metabolites are endogenous molecules produced in balance in healthy conditions. However, the actions of the metabolites depend on the environment or their concentrations and the activation of the pathway generally leads to unfavorable consequences

\section{The kynurenine system}

In the KYN system, tryptophan 2,3-dioxygenase (TDO), indoleamine 2,3-dioxygenases (IDOs), kynurenine aminotransferases (KATs), kynureninase (KYNU), and kynurenine 3-monooxygenase (KMO) are the main enzymes in the Try degradation cascade. Among them, TDO and IDOs are responsible for the rate-limiting step of L-Trp to L-KYN. The TDO is stimulated by the stress hormone cortisol, while IDOs are upregulated by lipopolysaccharides and pro-inflammatory cytokines, and downregulated by antioxidant enzyme superoxide dismutase and anti-inflammatory cytokines. The KATs catalyze the conversion of KYN to kynurenic acid (KYNA) and 3-hydroxy-L-kynurenine (3-HK) to xanthurenic acid (XA), while KYNU converts L-KYN to anthranilic acid (AA). The KMO converts L-KYN to 3-HK. This enzyme is stimulated by oxygen molecules and pro-inflammatory cytokines, while it is inhibited by superoxide dismutase and anti-inflammatory cytokines. Thus, the KYN enzymes are under the constant influence of the hypothalamicpituitary-adrenal axis, oxidative stress, the antioxidant system, and inflammatory responses. ${ }^{2,5-9}$

The 3-HK, 3-hydroxyanthranilic acid (3-HAA) and quinolinic acid (QA) are oxidants; however, 3-HK and
3-HAA may serve as antioxidants in certain conditions. Quinolinic acid stimulates glutamate receptors, while KYNA either stimulates or inhibits the glutamate receptors subgroups in a concentration-dependent manner. The L-KYN, KYNA, XA, and cinnabarinic acid (CA) are AHR agonists which relay the signal of immune tolerance. The AA serves as a water-soluble vitamin and its derivatives are nonsteroidal anti-inflammatory drugs such as diclofenac and mefenamic acid. Picolinic acid is thought to be a neuroprotectant (Fig. 1). The 3-HK stimulates TDO in vivo and NADH stimulates KMO, forming positive feedback loops; meanwhile, NADH inhibits TDO, forming a negative feedback loop within the KYN system. ${ }^{2,5-9}$

The molecules generated in the KYN system serve as components of and relay signals to the external biosystems: 3-HK, 3-HAA, and QA in the oxidative stress complex, 3-HK, 3-HAA, and KYNA in the antioxidant system, Trp in serotonin neurotransmission, QA and KYNA in glutamate neurotransmission, $\mathrm{XA}$ in the tetrahydrobiopterin pathway, KYNA and QA in the cannabinoid system, NADH in the NAD $\mathrm{NADH}^{+} \mathrm{Nedox}$ system, and KYN, KYNA, XA, and CA in AHR signaling. Thus, stressors in general lead to the activation of the KYN system, and the presence of the positive feedback loops further amplifies stress loads, imposing a heavy burden on a wide range of biosystems. ${ }^{2,5-9}$ 


\section{Monitoring the kynurenine system}

Measurements of KYN metabolites and their ratios have been taken from clinical samples of patients with neurodegenerative diseases and psychiatric disorders in search of a correlation with diseases and thus to identify possible biomarkers. ${ }^{2,10}$ Categorized analysis of neurotoxic KYNs and neuroprotective KYNs revealed higher levels of neurotoxic KYNs and higher ratios of neurotoxic KYNs in neurodegenerative and psychiatric diseases, but the levels and the ratios of neuroprotective KYNs remain unclear. $^{2,8,10}$ The levels of pro-inflammatory cytokines are invariably elevated in all diseases investigated, which therefore suggests that the elevation of neurotoxic KYNs is closely linked to the inflammatory response. ${ }^{2,8}$ Furthermore, low-grade inflammation (LGI) is considered to participate in the pathogenesis of neurodegenerative diseases, but the role of LGI in psychiatric disorders remains unclear. ${ }^{2}$ A meta-analysis of 101 studies of blood samples showed that decreased levels of Trp, KYN and KYNA, increased ratios of KYN/Trp, and decreased ratios of KYNA/KYN, KYNA/QA and KYNA/3-HK in patients with major depressive disorder (MDD), decreased levels of Trp and KYNA and decreased ratio of KYNA/ QA in patients with bipolar disorder (BD), and the decreased level of Trp in patients with schizophrenia. This study confirms the activation of the Trp-KYN pathway and depletion of Trp in 3 major psychiatric disorders and the increased shunt toward the production of toxic KYNs in MDD and BD. ${ }^{11}$ Further studies are expected to reveal the status of the KYN system and its interaction with functionally adjacent biosystems by assessing serotonin and glutamate neurotransmissions, tetrahydrobiopterin levels, antioxidant capacity, and immune tolerance.

\section{Homeostasis, allostasis, far-from- equilibrium, entropy}

The activation of the KYN system is at least partly a physiological reaction of the body to stressful events such as infection, inflammation, metabolic disturbance, aging, and anticipation, among others. The stability of le milieu intérieur (the internal environment) is essential to living independently of the external environment, as suggested by Claude Bernard, and homeostasis is the state within the physiological limits, proposed by Walter Cannon
(Fig. 2). ${ }^{12,13}$ Selye conceptualized the process in which chronically harmful stimuli lead to disease in the general adaptation syndrome. Organisms attempt to maintain homeostasis against harmful stimuli up to the resistance stage but eventually surrender to the stressors in the exhaustion stage. Stress is an organized state of bodies in response to stressors, experienced by all patients during a period of illness. Harmful consequences are not due to exhaustion of defense mechanism, but the stress mediators themselves cause damage to the host, especially in the exhaustion stage (Fig. 2). ${ }^{14}$ However, Selye's stress theory does not allow the measurements of stress or the evaluation of the reaction of systems under chronic stress.

Subsequently, Sterling and Eyer developed a concept of allostasis, which refers to the process of maintaining stability through change in the allostatic systems. The adaptational cost of chronic exposure to inappropriate response to the body, that is the allostatic load, eventually fails to perform normally, thus leading to diseases (Fig. 2) ${ }^{15}$ However, the allostatic concept stands on the vague relationship between metabolism and energy consumption, which makes it difficult to sample and measure allostatic loads, thus limiting the application of the concept for stress measurement.

Researchers in the field of thermodynamics have attempted to quantify homeostasis, stress and allostasis. Life feeds on negative entropy. The second law of thermodynamics states the entropy of an isolated system can only increase. The body is an open thermodynamic system. Order within the body is maintained by an increase in the disorder of the environment through dissipating heat from the body. ${ }^{16}$ It is supported in the nonequilibrium open system which prevails around us. The farfrom-equilibrium system does not lead to turbulence, but indeed, it forms life.$^{17}$ Entropy is directional with time and thus it can be applied to physiological growth and aging. ${ }^{18}$ A thermodynamic entropy-based model of the stress response has been proposed. Based on entropy production, the model describes the states of a living system under stress, and differentiates the states of health and disease by using measurable variables. Positive stress entropic load (SEL) leads to an adverse state, while negative SEL leads to a protective state of health. Furthermore, the model can predict not only the future failure of the living system but also the deterioration of the regulatory feedback (Fig. 2). ${ }^{19}$ Improving energy balance may become a possible strategy for disease intervention. ${ }^{20}$
1850
1926
1936
1988
2016
Walter Cannon
Hans Selye
Sterling \& Eyer
Bienertová-Vašků et al.

Claude Bernard

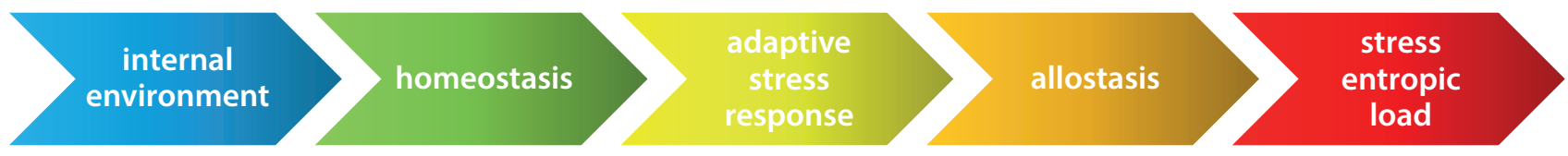

Fig. 2. Conceptual evolution of stress 


\section{Conclusions and future perspective}

Increasing amounts of evidence show the presence of KYN system activation in a wide range of diseases including neurodegenerative and psychiatric diseases. However, the levels and the ratios of KYN metabolites are equivocal among diseases and the actions of the metabolites are enigmatic depending on the environment and their concentrations. ${ }^{21}$ Furthermore, the KYN system varies from cell or tissue where some enzymes are missing, and different bioactive molecules are predominantly produced. ${ }^{10}$ Some metabolites can cross to the blood-brain barrier, but others cannot. ${ }^{10}$ Nevertheless, the KYN metabolites potentially serve as biomarkers and the KYN system may be one possible target for disease intervention. ${ }^{5,7,9,10}$ Very little is known about the interaction of the KYN system with adjacent biosystems. Understanding the interaction may open new diagnostic and interventional approaches toward various diseases.

Concepts of homeostasis, stress and allostasis have provided an intuitive insight into the action and effect of stress on the body and mind. Direct measurements of stressors, stress and the consequence remain far from feasible. Certainly, the KYN system is one of the major players under stress and in the pathogenesis of various diseases. Holistic approaches including understanding the interaction between other biosystems, theoretical reframing of the alteration of the KYN system under stress, and the application of empirical models to assess pathological processes and their consequences may provide a possible clue to understanding such a complex system.

The roles of the KYN system in physiological aging, the interaction of sex/gender factors, and nutritional and metabolic stress are of particular interest. ${ }^{22,23}$ Animal research will certainly make a solid contribution to the area. Transgenic and pharmacological animal models of cognitive, emotional and/or social impairment may reveal the roles of the aging process, and/or sex/gender factors in the pathogenesis of mental illnesses. Transgenic mice such as KYNA, KYNU or KMO knock-out strains and double knock-out strains may help understand the role of the KYN system in physiological aging, discover potential diagnostic biomarkers and search for possible interventional targets in neurodegenerative diseases and psychiatric disorders.

\section{ORCID iDs}

Masaru Tanaka (1) https://orcid.org/0000-0003-4383-4024 László Vécsei (1) https://orcid.org/0000-0001-8037-3672

\section{References}

1. Barik S. The uniqueness of tryptophan in biology: Properties, metabolism, interactions and localization in proteins. Int J Mol Sci. 2020; 21(22):8776. doi:10.3390/ijms21228776

2. Tanaka $M$, Tóth F, Polyák $H$, et al. Immune influencers in action: Metabolites and enzymes of the tryptophan-kynurenine metabolic pathway. Preprints. 2021;2021:2021060344. doi:10.20944/preprints 202106.0344.v1
3. van Donkelaar E, Blokland A, Ferrington L, et al. Mechanism of acute tryptophan depletion: Is it only serotonin? Mol Psychiatry. 2011;16(7): 695-713. doi:10.1038/mp.2011.9

4. Blankfield A. A brief historic overview of clinical disorders associated with tryptophan: The relevance to chronic fatigue syndrome (CFS) and fibromyalgia (FM). Int J Tryptophan Res. 2012;5:27-32. doi:10.4137/ IJTR.S10085

5. Tanaka M, Török N, Vécsei L. Editorial: Are 5-HT1 receptor agonists effective anti-migraine drugs? Expert Opin Pharmacother. 2021;12:1-5. doi:10.1080/14656566.2021.1910235

6. Encyclopedia. Available online: https://encyclopedia.pub/8633 (accessed on 21 June 2021).

7. Tanaka M, Bohár Z, Vécsei, L. Are kynurenines accomplices or principal villains in dementia? Maintenance of kynurenine metabolism. Molecules. 2020;25(3):564. doi:10.3390/molecules25030564

8. Tanaka M, Toldi J, Vécsei L. Exploring the etiological links behind neurodegenerative diseases: Inflammatory cytokines and bioactive kynurenines. Int J Mol Sci. 2020;21(7):2431. doi:10.3390/ijms21072431

9. Tanaka M, Török N, Vécsei L. Novel pharmaceutical approaches in dementia. Riederer P, Laux G, Nagatsu T, Le W, Riederer C, eds. NeuroPsychopharmacotherapy. Cham, Switzerland: Springer; 2021. doi:10.1007/978-3-319-56015-1_444-1

10. Török N, Tanaka M, Vécsei L. Searching for peripheral biomarkers in neurodegenerative diseases: The tryptophan-kynurenine metabolic pathway. Int J Mol Sci. 2020;21(24):9338. doi:10.3390/ijms21249338

11. MarxW, McGuinness AJ, Rocks T, et al. The kynurenine pathway in major depressive disorder, bipolar disorder, and schizophrenia: A meta-analysis of 101 studies [online ahead of print published on November 23, 2020]. Mol Psychiatry. 2020. doi:10.1038/s41380-020-00951-9

12. Bernard C. An Introduction to the Study of Experimental Medicine. Transl. H.C. Greene. New York, USA: Collier; 1865

13. Cannon WB. The Wisdom of the Body. New York, USA: Norton; 1932.

14. Selye H. The Stress of Life. $2^{\text {nd }}$ ed. New York, USA: McGraw-Hill; 1976.

15. Sterling $P$, Eyer J. Allostasis: A new paradigm to explain arousal pathology. In: Fisher S, Reason JT. Handbook of Life Stress, Cognition and Health. Chichester, USA: Wiley \& Sons; 1988.

16. Schrödinger E. What is Life? Cambridge, UK: Cambridge University Press; 1944.

17. Ornes S. Core Concept: How nonequilibrium thermodynamics speaks to the mystery of life. Proc Natl Acad Sci U S A. 2017;114(3):423-424. doi:10.1073/pnas.1620001114

18. Haddad WM. Thermodynamics: The unique universal science. Entropy. 2017;19:621. doi:10.3390/e19110621

19. Bienertová-Vašků J, Zlámal F, Nečesánek I, et al. Calculating stress: From entropy to a thermodynamic concept of health and disease. PLoS One. 2016:11(1):e0146667. doi:10.1371/journal.pone.0146667

20. Finger $E$. Thermodynamics as the driving principle behind the immune system. Einstein (Sao Paulo). 2012;10(3):386-388. doi:10.1590/ s1679-45082012000300024

21. Rózsa E, Robotka H, Vécsei L, Toldi J. The Janus-face kynurenic acid. J Neural Transm. 2008;115:1087-1091. https://doi.org/10.1007/s00702008-0052-5

22. Muntsant A, Jiménez-Altayó F, Puertas-Umbert L, et al. Sex-dependent end-of-life mental and vascular scenarios for compensatory mechanisms in mice with normal and AD-neurodegenerative aging. Biomedicines. 2021;9(2):111. doi:10.3390/biomedicines9020111

23. Giménez-Llort L, Marin-Pardo D, Marazuela P, Hernández-Guillamón M. Survival bias and crosstalk between chronological and behavioral age: Age- and genotype-sensitivity tests define behavioral signatures in middle-aged, old and long-lived mice with normal and AD-associated aging. Biomedicines. 2021;9(6):636. doi:10.3390/ biomedicines 9060636

24. Kordestani MoghadamP, Nouriyengejeh S, Seyedhoseini B, Pourabbasi A. The study of relationship between nutritional behaviors and metabolic indices: A systematic review. Adv Biomed Res. 2020;9:66. doi:10. 4103/abr.abr_12_20

25. Kordestani-Moghadam P, Assari S, Nouriyengejeh S, Mohammadipour F, Pourabbasi A. Cognitive impairments and associated structural brain changes in metabolic syndrome and implications of neurocognitive intervention. J Obes Metab Syndr. 2020;29(3):174-179. doi:10.7570/jomes20021 